\title{
VALORACIÓN DE LA APTITUD FÍSICA EN NIÑOS Y ADOLESCENTES: CONSTRUCCIÓN DE CARTAS PERCENTÍLICAS PARA LA REGIÓN CENTRAL DEL PERÚ
}

\author{
Alcibíades Bustamante ${ }^{1,2, a}$, Gastón Beunen ${ }^{3, b \dagger}, J^{\prime}$ sé Maia ${ }^{1, b}$
}

\begin{abstract}
RESUMEN
Objetivos. Establecer cartas percentílicas y valores de referencia estratificada por edad y sexo de los niveles de aptitud física (AF) en niños y adolescentes de la región central del Perú. Materiales y métodos. El tamaño de la muestra comprendió a 7843 escolares (4155 mujeres y 3688 varones) entre los seis y los diecisiete años de edad. Los niveles de aptitud física fueron evaluados mediante el uso de seis pruebas motoras provenientes de las baterías EUROFIT, FITNESSGRAM y AAPHERD. Las cartas percentílicas fueron construidas por separado para cada sexo, utilizando el método matemático LMS implementado en el programa LMSchartmaker. Resultados. Se verifica valores superiores de AF en los varones, a excepción de la prueba de flexibilidad; la AF incrementa con la edad. Conclusiones. Existe variabilidad interindividual en ambos sexos. Los valores de referencia específicos por edad y sexo pueden utilizarse para la evaluación e interpretación de los niveles de AF de niños y adolescentes de la región central del Perú. Estos hallazgos pueden ayudar en la evaluación de programas de educación física en las escuelas.
\end{abstract}

Palabras clave: Rendimiento deportivo; Estructura corporal; Actividad motora; Niño; Adolescente (fuente: DeCS BIREME).

\section{EVALUATION OF PHYSICAL FITNESS LEVELS IN CHILDREN AND ADOLESCENTS: ESTABLISHING PERCENTILE CHARTS FOR THE CENTRAL REGION OF PERU}

\begin{abstract}
Objectives. Construct percentile charts and physical fitness (PF) reference values stratified by age and sex of children and adolescents from Peru's central region. Materials and methods. The sample was comprised of 7,843 subjects $(4,155$ females and 3,688 males) between the ages of 6 to 17 years old. Physical fitness was assessed using six tests developed by EUROFIT, FITNESSGRAM and AAPHERD. Percentile charts were developed separately for males and females using the LMS method calculated with LMSchartmaker software. Results. Males showed higher PF values with the exception of flexibility; a clear increase in PF with increasing age was verified. Conclusions. Inter-individual variability in both sexes is substantial. Charts and specific reference values by age and sex may be used for the assessment and interpretation of children's and adolescents' PF levels in Peru's central region. These findings may be of help to educators, public health professionals, parents, and policy-makers when assessing schools' physical education programs.
\end{abstract}

Key words: Athletic performance; Somatotypes; Motor activity; Child; Adolescent (source: MeSH NLM).

\section{INTRODUCCIÓN}

A finales de la década de 1970 emerge y se desarrolla el concepto de aptitud física orientada a la salud, asumiendo como propósito principal la discusión de aspectos esenciales del bienestar del individuo por encima del objetivo tradicional orientado al rendimiento deportivo ${ }^{(1,2)}$. El concepto de aptitud física (AF) se encuentra aún en pleno desarrollo, siendo considerada como una medida integrada de funciones y estructuras corporales (morfológica, muscular, motora, cardiorrespiratoria y metabólica) ${ }^{(3)}$ que pueden variar o mantenerse a lo largo del tiempo, reflejando de esta manera su carácter estático y dinámico ${ }^{(4)}$.

Los niveles de AF están determinados por un conjunto de factores, siendo los factores genéticos (que se manifiestan básicamente en los procesos de crecimiento, desarrollo y maduración) responsables del $40 \%$ o más de la variación total de la AF. Otro factor importante es el factor ambiental que incluye la actividad física (entendida como cualquier movimiento corporal producido por los músculos esqueléticos que resulte en gasto calórico) ${ }^{(5)}$ y el ejercicio físico (considerado

\footnotetext{
Laboratorio de Cineantropometria y Estadística Aplicada, Facultad de Deporte de la Universidad de Porto. Porto, Portugal.

Universidad Nacional de Educación Enrique Guzmán y Valle. Lima, Perú.

Facultad de Kinesiología y Ciencias de Rehabilitación, Universidad Católica de Lovaina. Brabante Valón, Bélgica.

Licenciado en Educación Física magíster en Desarrollo Motor; ${ }^{\text {b }}$ licenciado en Educación Física doctor en Ciencias del Deporte

$\dagger$ Fallecido
}

Recibido: 03-04-12 Aprobado: 30-05-12 
como la actividad física planificada, estructurada, sistematizada y deliberada) ${ }^{(6)}$.

Estudios recientes han puesto de manifiesto que niveles moderados a vigorosos de ejercicio físico y actividad física presentan un efecto protector para las enfermedades no transmisibles que son las causas más comunes de morbilidad y mortalidad en adultos ${ }^{(7)}$, y son un potente indicador del estado de salud de niños y adolescentes ${ }^{(6,8)}$. La AF en la niñez o adolescencia condiciona el nivel de AF y la presencia de factores de riesgo cardiovascular convencionales en la vida adulta ${ }^{(9,10)}$. De modo que, moderados o elevados índices cardiorrespiratorios, de fuerza/resistencia muscular, flexibilidad, así como niveles bajos de grasa corporal que son componentes de la AF relacionada con la salud, están asociados con un menor riesgo para el desarrollo de enfermedades degenerativas.

Existen varios estudios (2,11-14), incluidos estudios en Sudamérica ${ }^{(15-17)}$, que brindan valores de referencia en $A F$ para poblaciones específicas, orientando su utilización en el campo educativo y de la salud pública. En este contexto, la evaluación normativa permite establecer una distribución percentílica del comportamiento de los valores relativos a los componentes de la aptitud física y, a partir de ella, representarlos en cartas percentílicas. Estas cartas permiten detallar valores de referencia para una población específica, posicionar a cada niño o adolescente en términos percentílicos relativamente a la población estudiada ${ }^{(2,12)}$.

El noveno propósito establecido en el Diseño Curricular Nacional de Educación Básica Regular formulado por el Ministerio de Educación del Perú, se refiere al desarrollo corporal, y conservación de la salud física y mental, en el Área Curricular de Educación Física correspondiente a los niveles educativos de primaria y secundaria; sin embargo, no se presentan valores de referencia que permitan evaluar los niveles de AF en escolares peruanos ${ }^{(18)}$. Además, los valores normativos de países desarrollados no tienen correspondencia en los de países en desarrollo por razones económicas, sociales, culturales y geográficas.

Esta situación conlleva a la necesidad de proveer a los profesionales de la educación y la salud pública, así como a los padres de familia y autoridades políticas peruanas, valores de referencia sobre los niveles de AF de escolares pertenecientes a diferentes contextos. Por ello, el objetivo del presente trabajo es establecer los valores de referencia de AF, estratificada por edad y sexo, en escolares peruanos de la región central del Perú.

\section{MATERIALES Y MÉTODOS}

\section{TIPO Y POBLACIÓN DE ESTUDIO}

El presente trabajo forma parte del Proyecto Crecer con Salud y Esperanza (Resolución 2459-R-2008UNE); que evalúa crecimiento, composición corporal, desempeño motor, maduración biológica y actividad física en escolares de enseñanza básica y familias nucleares peruanas (efectos genéticos, socioculturales, geográficos y sus interacciones). Se trata de un estudio descriptivo y transversal realizado en niños y adolescentes en edad escolar entre 3 y 17 años de edad, residentes en cuatro distritos ubicados en regiones geográficamente diferenciadas en la zona central del Perú: en la costa, el distrito de Barranco (50 metros de altitud), que forma parte de la provincia de Lima; en la sierra, el distrito de Junín (4130 metros de altitud), perteneciente a la provincia del mismo nombre y, en la selva, los distritos de La Merced y San Ramón (700 y 750 metros de altitud) que presentan una continuidad geográfica e integran la provincia de Chanchamayo.

Con base en la información obtenida del Ministerio de Educación (19), referente a la distribución de los escolares matriculados en los años 2009 y 2010, se decidió seleccionar una muestra estratificada por escuelas públicas, y en cada escuela fue efectuada una selección aleatoria de los escolares. Así, de 31 instituciones educativas públicas localizadas en zona urbana, trece pertenecían al nivel inicial, diez al primario y ocho al secundario. Se evaluó a un 38\% del total de escolares matriculados (Tabla 1). Según el tipo de escuela donde cursaban estudios, los escolares pertenecían al nivel socioeconómico medio; se eligió este nivel para facilitar su comparación con otros países en vías de desarrollo ${ }^{(20)}$.

Se estableció el siguiente criterio de exclusión: diagnóstico clínico de discapacidad física, sensorial e intelectual indicado en el informe médico de cada participante. Esta exclusión se realizó a posteriori (1,2\%), sin conocimiento del alumno, respetando la dignidad y confidencialidad de los participantes.

Tabla 1. Porcentaje de escolares evaluados en función del total de matriculados en cada distrito.

\begin{tabular}{|c|c|c|c|c|c|}
\hline \multirow[b]{2}{*}{ Distrito } & \multicolumn{2}{|c|}{ Matriculados } & \multicolumn{3}{|c|}{ Evaluados } \\
\hline & Total & $\begin{array}{l}\text { Solo IE } \\
\text { públicas }\end{array}$ & Total & $\begin{array}{c}\% \\
\text { Total }\end{array}$ & $\begin{array}{c}\% \text { IE } \\
\text { públicas }\end{array}$ \\
\hline Barranco (costa) & 16448 & 10194 & 3169 & 19,3 & 31,1 \\
\hline Junín (sierra) & 3534 & 3306 & 2126 & 60,2 & 64,3 \\
\hline $\begin{array}{l}\text { La Merced - San } \\
\text { Ramón (selva) }\end{array}$ & 14097 & 12111 & 4494 & 31,9 & 37,1 \\
\hline TOTAL & 34079 & 25611 & 9789 & 28,7 & 38,2 \\
\hline
\end{tabular}

* IE: institución educativa 
La edad se calculó restando la fecha de evaluación y la fecha de nacimiento. Cada grupo de edad se construyó considerándose la edad inferior en 0,00 y la edad superior 0,99; por ejemplo, los niños cuyas edades decimales se encuentran entre 6,00 y 6,99 pertenecen al grupo de edad de 6 años de edad.

\section{EVALUACIÓN DE LA APTITUD FÍSICA}

Se aplicaron en total seis pruebas de AF. Cuatro pruebas de la batería de Aptitud Física Europea EUROFIT (21), para evaluar los niveles de fuerza muscular estática y explosiva; flexibilidad y velocidad/agilidad; la prueba de curl up de la batería Fitnessgram ${ }^{(22)}$ para medir la resistencia muscular; y la prueba de correr/caminar de doce minutos de la batería AAPHERD (11) para evaluar la resistencia cardiorrespiratoria. Estas pruebas se describen a continuación:

1. Flexión de tronco adelante en posición sentado. Con el escolar sentado en el suelo y valiéndose de un soporte de madera estandarizado ${ }^{(21)}$, se determinó la máxima distancia alcanzada con la punta de los dedos medios mediante la flexión anterior del tronco. Esta prueba mide la amplitud de movimiento o flexibilidad.

2. Dinamometría manual. Con el empleo de un dinamómetro digital Takei TKK 5401 se valoró la fuerza de prensión máxima en ambas manos.

3. Salto horizontal con pies juntos y sin carrera preparatoria. Con los pies paralelos detrás de una línea, rodillas semiflexionadas. A una señal el alumno deberá saltar la distancia máxima posible. Esta prueba determina la fuerza explosiva de las extremidades inferiores.

4. Carrera de ida y vuelta 10 x 5. Se evalúa de manera integrada la velocidad de desplazamiento y la agilidad. Cada escolar realiza cinco ciclos (ida y vuelta) a la máxima velocidad entre dos líneas separadas a cinco metros.

5. Fuerza abdominal (curl up). Realizado según los parámetros establecidos para esta prueba (22). Decúbito dorsal con rodillas flexionadas y los brazos extendidos a lo largo del cuerpo. Se registró el número de elevaciones y descensos del tronco realizados correctamente hasta un límite de 75 . Mide la fuerza y la resistencia de la musculatura abdominal.

6. Correr/caminar de 12 minutos. En campo delimitado previamente, los escolares van corriendo o
Tabla 2. Estimación de fiabilidad: valores del coeficiente de correlación intraclase.

\begin{tabular}{lccc}
\hline Pruebas motoras & $\mathbf{n}$ & $\mathbf{C C l}$ & $\mathbf{I C}$ 95\% \\
\hline Prensión & 211 & 0,96 & $0,94-0,97$ \\
Curl up & 211 & 0,91 & $0,88-0,93$ \\
Salto horizontal & 211 & 0,98 & $0,97-0,98$ \\
Flexibilidad & 211 & 0,89 & $0,86-0,92$ \\
Va-viene 10 x 5 & 210 & 0,88 & $0,85-0,91$ \\
Correr/caminar 12" & 125 & 0,87 & $0,82-0,91$ \\
\hline
\end{tabular}

* IC 95\%: intervalos de confianza al 95\%

caminando el máximo de distancia posible en doce minutos. Prueba que valora la aptitud cardiorrespiratoria.

El equipo de evaluadores estuvo constituida por seis profesores y veinticinco estudiantes de Educación Física de la UNE EGyV, quienes formaron parte de un programa de entrenamiento sobre los procedimientos técnicos en la administración de las pruebas motoras. La evaluación de los escolares se realizó entre marzo de 2009 y junio de 2011 en horario escolar, en instalaciones deportivas facilitadas por las municipalidades de Chanchamayo y Junín; así como en los espacios deportivos de las IE de San Ramón y Barranco.

\section{CONTROL DE CALIDAD DE LA INFORMACIÓN}

Se realizó en dos fases: la primera correspondió al estudio piloto realizado en las instalaciones de la UNE EGyV en marzo de 2009 y una segunda donde se utilizó el procedimiento reliability in field, para lo cual se aplicó un retest a tres o cinco escolares elegidos aleatoriamente en días alternos de evaluación. Los instrumentos (cronómetros, cintas, etc.) fueron revisados y calibrados al inicio de cada día de evaluación.

Además, se determinó al coeficiente de correlación intraclase para cada prueba. Los valores obtenidos indican una alta fiabilidad, por lo que el grado de consistencia con que se registran u observan valores semejantes en dos puntos de tiempo es alto. En la Tabla 2 se presentan los resultados del $\mathrm{CCl}$ referidos a las estimaciones puntuales y de intervalos de confianza al 95\% (IC 95\%).

\section{ANÁLISIS ESTADÍSTICO}

Se efectuaron pruebas de normalidad mediante las pruebas de Kolmogorov-Smirnov; para estudiar el efecto de la edad y del sexo en las pruebas de aptitud física se utilizó el análisis de la variancia a dos factores (ANOVA II), así como se determinó la fiabilidad 
A

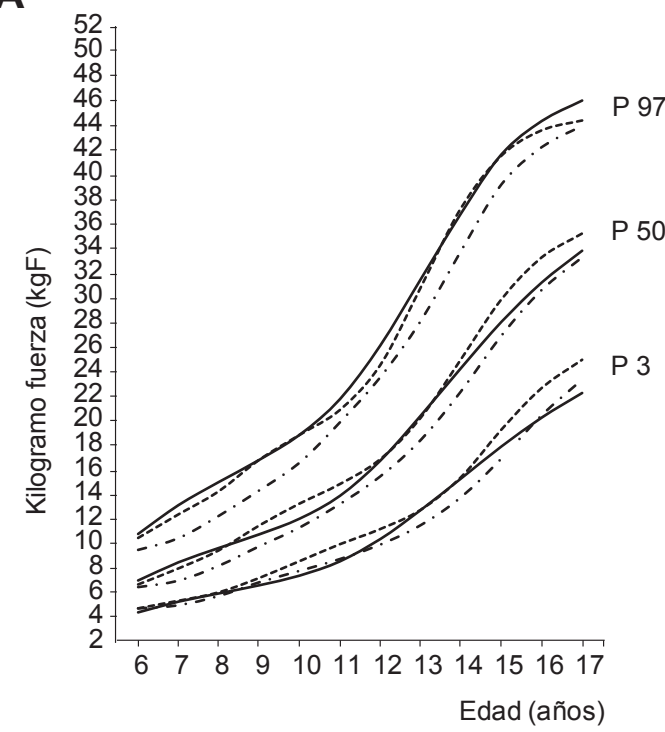

B

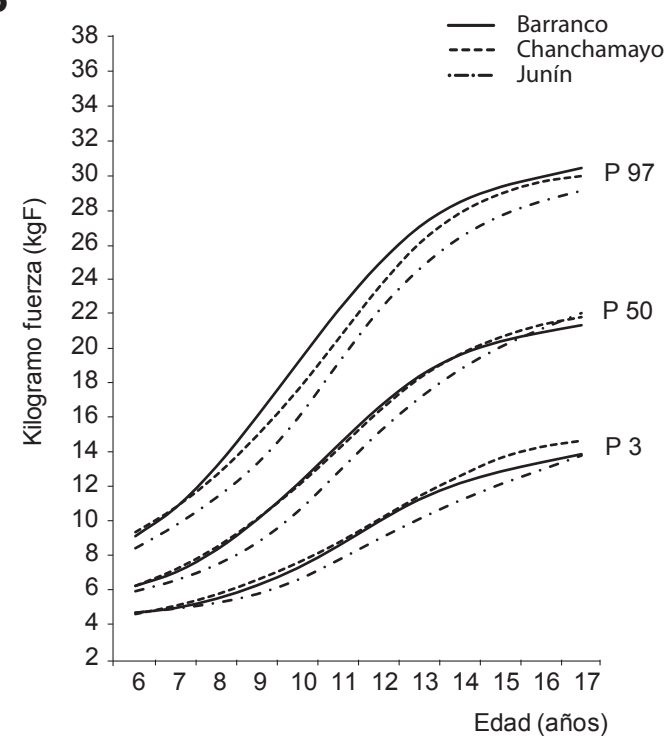

Figura 1. Comportamiento de los valores de la mediana (P50) y de los percentiles extremos (P3 y P97) de la fuerza de presión según procedencia. A) Masculino; B) Femenino.

test-retest para las pruebas motoras. El análisis de los datos se realizó en el programa estadístico IBM SPSS Statistics versión 20 .

Para determinar los valores percentílicos y luego construir sus respectivas cartas para las pruebas de aptitud física para cada sexo se recurrió al método del LMS propuesto por Cole \& Green (23) implementado en el programa LMSchartmaker Pro versión $2.54^{(24)}$. El método LMS permite que la distribución de datos independientes con valores positivos pueda ser normalizada en cada edad recurriendo a la transformación de Box-Cox. Son producidas tres curvas suavizadas y específicas para cada edad: L, M y S, donde la curva L (transformación Box-Cox), curva M (mediana), y curva S (coeficiente de variación), en base a la siguiente ecuación:

$$
C_{100 \alpha}(t)=M(t)[1+L(t) S(t) Z \alpha]^{1 / L(t)}
$$

Donde, Za es el desvío normal equivalente para la muestra total, $\alpha$ y $C_{100 \alpha}(t)$ el percentil que corresponde. Los grados de libertad equivalentes para $L(t), M(t)$ y $S(t)$ miden la complejidad del alineamiento de cada curva. El número apropiado de los grados de libertad y calidad de los modelos fue determinado de acuerdo con las sugerencias de Pan y Cole ${ }^{25)}$, basados en el valor de la Deviance ${ }^{(26)}$ y en las representaciones gráficas del Q-test y Worm-plots ${ }^{(27,28)}$.

Anterior a la obtención de los valores percentílicos y construcción de las cartas de referencia, se calculó los valores porcentuales y se elaboró curvas para los percentiles $\mathrm{P}_{3} ; \mathrm{P}_{50}$ y $\mathrm{P}_{97}$ con la finalidad de verificar la variabilidad de los valores correspondiente a cada prueba motora en cada grupo de edad, sexo y distrito de residencia. Esta variabilidad presumida y constatada posteriormente justifica el procesamiento en conjunto de la totalidad de la información recogida según grupo de edad, sexo y prueba motora.

\section{CONSIDERACIONES ÉTICAS}

El Proyecto Crecer con Salud y Esperanza fue aprobado por el Instituto de Investigación de la Universidad Nacional de Educación Enrique Guzmán y Valle (UNE EGyV). El estudio se realizó siguiendo las pautas éticas de la Declaración de Helsinki 1961 (revisión de Edinburgh 2000). Se solicitó la autorización de los padres o tutores y el consentimiento individual de cada niño y adolescente, además del permiso otorgado por autoridades de las escuelas participantes en el estudio; manteniendo la confidencialidad de los datos.

\section{RESULTADOS}

La muestra total estuvo compuesta por 7843 escolares, de los cuales 4155 fueron mujeres y 3688 varones, con edades comprendidas entre 6 y los 17 años (Tabla 3). 
Tabla 3. Distribución de la muestra por distrito, edad y sexo.

\begin{tabular}{ccc} 
Barranco Junín & $\begin{array}{c}\text { Chancha- } \\
\text { mayo }\end{array}$ & Total \\
\hline
\end{tabular}

Edad Fem. Masc. Fem. Masc. Fem. Masc. Fem. Masc.

\begin{tabular}{rrrrrrrrr}
\hline 6 & 99 & 70 & 43 & 48 & 156 & 157 & 298 & 275 \\
7 & 86 & 62 & 50 & 47 & 150 & 192 & 286 & 301 \\
8 & 82 & 64 & 47 & 60 & 178 & 174 & 307 & 298 \\
\hline 9 & 119 & 76 & 65 & 76 & 187 & 184 & 371 & 336 \\
10 & 85 & 85 & 70 & 71 & 218 & 190 & 373 & 346 \\
\hline 11 & 111 & 114 & 73 & 55 & 194 & 191 & 378 & 360 \\
\hline 12 & 92 & 119 & 123 & 90 & 237 & 144 & 452 & 353 \\
\hline 13 & 83 & 64 & 93 & 92 & 212 & 144 & 388 & 300 \\
\hline 14 & 111 & 102 & 103 & 102 & 187 & 120 & 401 & 324 \\
\hline 15 & 110 & 127 & 98 & 84 & 150 & 140 & 358 & 351 \\
16 & 128 & 86 & 100 & 78 & 126 & 116 & 354 & 280 \\
\hline 17 & 73 & 51 & 63 & 52 & 53 & 61 & 189 & 164 \\
\hline Total & 1179 & 1020 & 928 & 855 & 2048 & 1813 & 4155 & 3688 \\
\hline
\end{tabular}

En las Figuras 2 y 3 se presentan las curvas percentílicas suavizadas $\left(\mathrm{P}_{3} ; \mathrm{P}_{10} ; \mathrm{P}_{25} ; \mathrm{P}_{50} ; \mathrm{P}_{75} ; \mathrm{P}_{90}\right.$ y $\left.\mathrm{P}_{97}\right)$ de las seis pruebas de aptitud física para ambos sexos, y en función de la edad. Los resultados demuestran una mayor aptitud física por parte de los varones, niños y adolescentes, con excepción de la prueba de flexibilidad, en la cual las mujeres manifiestan un mejor desempeño.

El perfil de las curvas percentílicas que corresponde a los varones presenta una tendencia al incremento de los niveles de aptitud física a lo largo de la edad, mientras que las mujeres mostraron una mayor estabilidad y un menor incremento.

Las diferencias en cada prueba de AF en relación a la edad y sexo fue significativa $(p<0,001)$. En la Tabla 4 se muestran los valores para los escolares, se verifica un efecto claro de la edad en todas las pruebas motoras, que evidencian aumentos de las medias a medida que avanza la edad. Los escolares varones presentan en cinco de las pruebas motoras valores medios más elevados en casi todos los intervalos de edad, a excepción de la prueba de flexibilidad. La interacción entre edad y sexo fue significativa en todas las pruebas motoras estudiadas.

En las Figuras 2 y 3 puede verificarse que los varones, por lo general, fueron más homogéneos en tres de las pruebas (dinamometría, curl up y salto horizontal) y las mujeres en las restantes (flexibilidad, agilidad y resistencia cardiorrespiratoria). La información disponible en el Anexo (www.rpmesp.ins.gob.pe) muestran los valores normativos expresados en percentiles del $\mathrm{P}_{3}$ al $\mathrm{P}_{97}$ de la aptitud física estratificada por edad y sexo de niños y adolescentes peruanos, que complementa esta información y muestra, por ejemplo, que la amplitud percentílica entre $\mathrm{P}_{3}$ y $\mathrm{P}_{97}$ en la prueba de salto horizontal fue de 96,6 y $67,8 \mathrm{~cm}$ en varones y mujeres, respectivamente.

Tabla 4. Valores descriptivos en pruebas de aptitud física en escolares de la región central del Perú.

\begin{tabular}{|c|c|c|c|c|c|c|}
\hline \multirow[b]{2}{*}{ Prueba* } & \multicolumn{6}{|c|}{ Edad (años) } \\
\hline & 6 & 8 & 10 & 12 & 14 & 16 \\
\hline \multicolumn{7}{|c|}{$\overline{\text { Prensión (kgF) }}$} \\
\hline Femenino & $6,7 \pm 1,2$ & $9,3 \pm 2,2$ & $13,2 \pm 2,9$ & $17,4 \pm 3,5$ & $19,9 \pm 4,0$ & $21,4 \pm 4,0$ \\
\hline Masculino & $7,5 \pm 1,6$ & $10,2 \pm 2,5$ & $13,6 \pm 2,6$ & $18,1 \pm 4,0$ & $26,7 \pm 5,5$ & $33,5 \pm 5,2$ \\
\hline \multicolumn{7}{|c|}{ Fuerza y resistencia abdominal (repeticiones) } \\
\hline Femenino & $21,9 \pm 11,9$ & $29,7 \pm 12,8$ & $35,2 \pm 15,1$ & $36,5 \pm 16,6$ & $34,8 \pm 15,2$ & $38,6 \pm 13,7$ \\
\hline Masculino & $22,3 \pm 10,9$ & $32,5 \pm 13,5$ & $41,0 \pm 17,8$ & $48,2 \pm 18,8$ & $53,8 \pm 18,3$ & $51,7 \pm 17,9$ \\
\hline \multicolumn{7}{|c|}{ Salto horizontal (cm) } \\
\hline Femenino & $83,5 \pm 14,4$ & $104,7 \pm 17,0$ & $117,9 \pm 18,2$ & $123,2 \pm 17,4$ & $121,0 \pm 17,7$ & $122,6 \pm 16,1$ \\
\hline Masculino & $95,6 \pm 15,6$ & $112,7 \pm 18,3$ & $124,4 \pm 18,0$ & $132,9 \pm 19,4$ & $150,3 \pm 23,2$ & $160,2 \pm 23,9$ \\
\hline \multicolumn{7}{|c|}{ Flexibilidad (cm) } \\
\hline Femenino & $23,8 \pm 3,2$ & $22,8 \pm 3,7$ & $21,5 \pm 4,5$ & $22,2 \pm 4,3$ & $22,6 \pm 4,0$ & $23,8 \pm 4,6$ \\
\hline Masculino & $22,4 \pm 4,0$ & $22,0 \pm 3,8$ & $20,5 \pm 3,4$ & $20,1 \pm 3,6$ & $21,7 \pm 3,7$ & $23,4 \pm 3,4$ \\
\hline \multicolumn{7}{|c|}{ Agilidad (s) } \\
\hline Femenino & $27,3 \pm 2,4$ & $25,3 \pm 2,5$ & $24,2 \pm 2,5$ & $24,2 \pm 2,2$ & $24,2 \pm 2,1$ & $23,5 \pm 1,8$ \\
\hline Masculino & $26,2 \pm 2,1$ & $24,2 \pm 2,6$ & $22,7 \pm 2,4$ & $22,0 \pm 2,5$ & $21,1 \pm 1,8$ & $20,2 \pm 1,4$ \\
\hline \multicolumn{7}{|c|}{ Correr/caminar $12 \min (\mathrm{m})$} \\
\hline Femenino & $1091,1 \pm 219,4$ & $1311,3 \pm 248,4$ & $1423,9 \pm 294,2$ & $1408,4 \pm 283,1$ & $1450,1 \pm 296,5$ & $1485,6 \pm 271,8$ \\
\hline Masculino & $1568,0 \pm 131,6$ & $1622,3 \pm 229,8$ & $1606,8 \pm 239,7$ & $1685,8 \pm 220,9$ & $1794,4 \pm 180,0$ & $1948,9 \pm 213,4$ \\
\hline
\end{tabular}




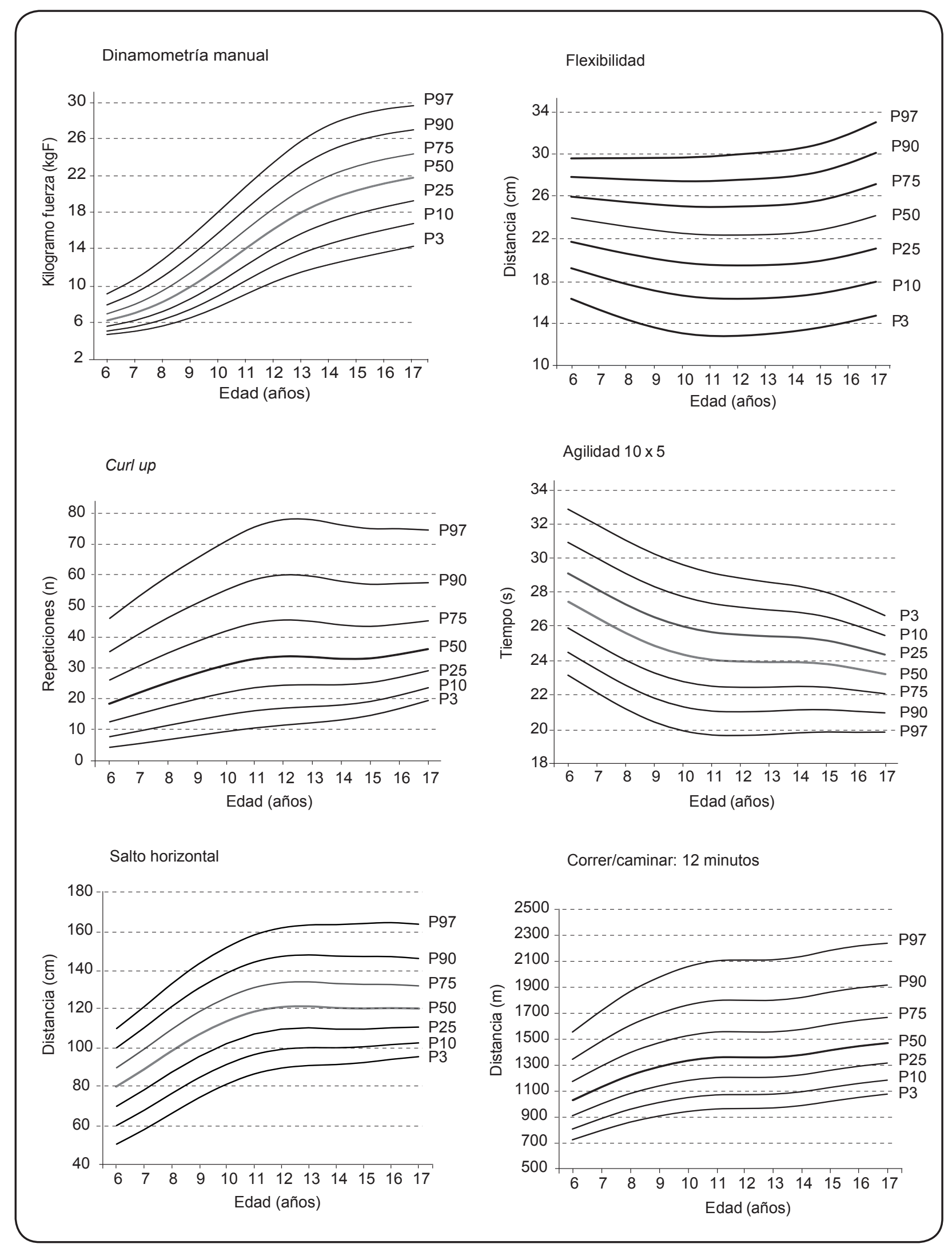

Figura 2. Cartas percentílicas de aptitud física en niñas y adolescentes. 

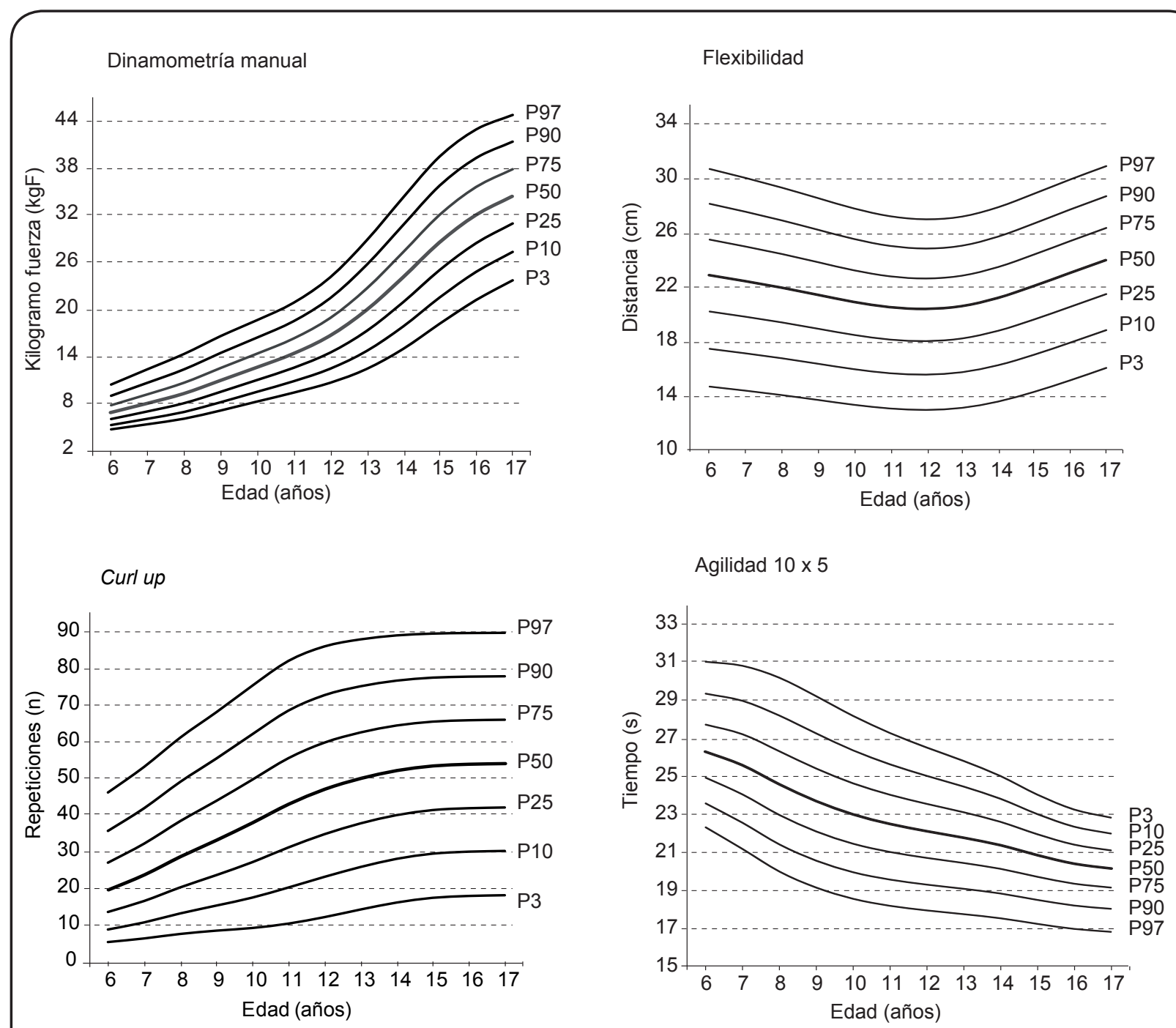

Salto horizontal

Correr/caminar: 12 minutos
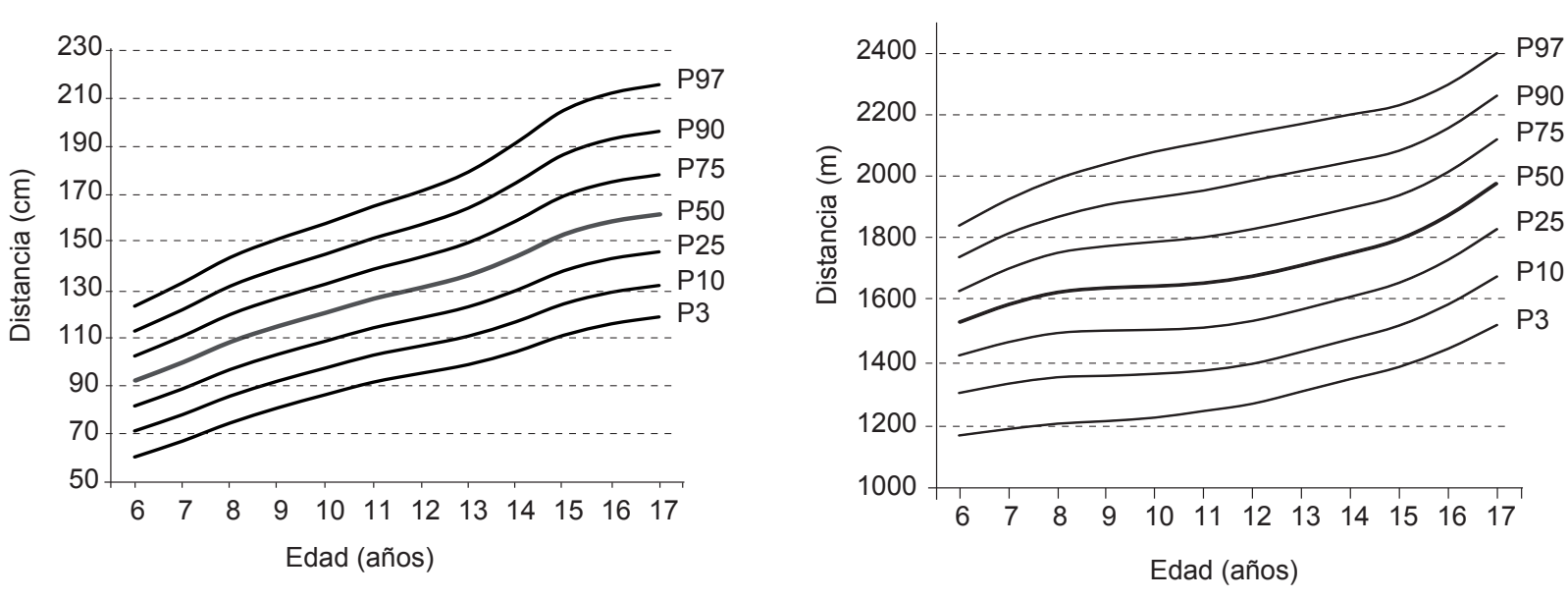

Figura 3. Cartas percentílicas de aptitud física en niños y adolescentes. 


\section{DISCUSIÓN}

Este estudio presenta los valores de AF en forma de cartas percentílicas específicas por sexo y edad para seis pruebas de AF en niños y adolescentes provenientes de escuelas públicas de la región central del Perú. Una interpretación apropiada de la evaluación de los niveles de $A F$ requiere que los valores encontrados en una persona sean comparados con valores de referencia de la población para el mismo sexo y edad; de modo que estos valores pueden utilizarse como datos normativos y también sirvan como valores de línea de base para el posterior seguimiento de los niveles de AF de los niños y adolescentes peruanos.

Los valores de referencia para una población deben reunir consistencia y rigurosidad en el proceso de recolección de la información y respetar principios establecidos para su construcción. Los resultados correspondientes a la fiabilidad encontrados en la evaluación de los diferentes componentes de la aptitud física revelan estimativas elevadas, las cuales van de 0,87 en la prueba de correr/ caminar de doce minutos a 0,98 en salto horizontal. Estos resultados son similares a los encontrados en otros estudios como la de Freitas ${ }^{(2)}$ y Silva ${ }^{(15)}$ que varían entre 0,70 a 0,98 y 0,87 a 0,98 , respectivamente. En cuanto a la validez, la revisión sistemática de literatura, los diferentes estudios que hicieron uso de ellas, así como el proceso sistemático de construcción de las baterías explicadas en sus respectivos manuales ${ }^{(2,11,12,21-22)}$ de las cuales se tomó las seis pruebas, aseguran su validez y aplicabilidad. También durante el proceso de recolección se controló el factor "aprendizaje" y el "agotamiento" evitando repeticiones innecesarias; por otro lado, se motivó permanentemente a cada participante con la finalidad de que pueda brindar su mayor esfuerzo en la ejecución de cada prueba.

Destaca que los varones muestran valores medios más elevados en cinco de las pruebas, y en la mayoría de los intervalos de edad. Las mujeres presentan valores medios superiores en la prueba de flexibilidad, y a partir de los 11-12 años en las demás pruebas presentan una estabilización o declinación de su nivel de aptitud física. Estos resultados corroboran comportamiento similar a otros estudios realizados ${ }^{(2,12-15)}$.

Las Figuras 2 y 3 muestran las curvas para las pruebas de AF según sexo. Se verifica incrementos a lo largo de la edad en las pruebas motoras en ambos sexos; en los varones aumentan linealmente de los seis a los diecisiete años en las pruebas de prensión y salto horizontal, observándose mayores incrementos a partir de los 12-13 años de edad en la primera prueba, mientras que en las mujeres los incrementos son graduales a lo largo de la edad sin señales de un "salto" en la prueba de prensión. En la prueba de salto horizontal, los incrementos lineales se presentan hasta los trece años en las mujeres, a partir de la cual existe una tendencia a estabilizarse y disminuir gradualmente. La fuerza abdominal que es evaluada a partir de la prueba de curl up mejoran linealmente con la edad hasta los 1213 años en las mujeres, seguido de una "meseta" hasta los diecisiete años. En los varones estos incrementos se presentan hasta los trece años, momento a partir del cual los valores percentílicos tienden a estabilizarse. En esta prueba se observa una mayor variación interindividual en cada edad y en ambos sexos semejante a los hallados en otros estudios ${ }^{(12,15,29)}$.

Comportamientos similares en las pruebas de dinamometría manual y salto horizontal se verificaron en estudios realizados en Portugal ${ }^{(2,12)}$ y Brasil (15). El desarrollo de la fuerza muscular progresa con la edad; sin embargo, el patrón de mejora no es uniforme en las diferentes pruebas motoras. Malina et al. (1) refieren, en relación con la fuerza estática, que en los varones se verifica una aceleración del desarrollo de la fuerza a partir de los trece años, que coincide con el estirón del crecimiento en la adolescencia, mientras que en las mujeres no se observa con nitidez este comportamiento. Asimismo, señala que debe tenerse en cuenta la importancia de la maduración del tejido muscular en el desarrollo de la fuerza y en la mejora del desempeño.

La flexibilidad en las mujeres decrece con la edad hasta los once años, se estabiliza, y luego aumenta hasta los diecisiete años; mientras que en los varones el decrecimiento de sus valores se da hasta los doce años y su posterior incremento es más acentuado. Las diferencias en los valores del $\mathrm{P}_{50}$ son más elevadas entre los diez y los trece años en las mujeres en relación con los varones $(2 \mathrm{~cm})$. Este comportamiento es similar a lo encontrado para esta prueba en el estudio realizado en Madeira ${ }^{(2)}$ y Letonia ${ }^{(30)}$. Sin embargo, los incrementos entre los seis y los diecisiete años en cada percentil, es bajo en ambos sexos. Este patrón de comportamiento de los valores es atribuido, en parte, al crecimiento de las extremidades y del tronco durante la adolescencia ${ }^{(1)}$.

La carrera de va-viene $10 \times 5$ es un indicador de agilidad y velocidad. Se verifica una disminución acentuada del tiempo de la prueba a medida que la edad aumenta hasta los diecisiete años en los varones. En las mujeres, esta mejora del desempeño es hasta los once años, a partir de la cual se estabiliza en los siguientes cuatro grupos de edad, para luego volver a mejorar en los percentiles $\mathrm{P}_{3}$ al $\mathrm{P}_{50}$, mientras que en las restantes se estabiliza hasta los diecisiete años. Los varones presentan mejor desempeño en 
relación con las mujeres en todos los intervalos de edad. Los resultados son semejantes en ambos sexos a lo descrito en los estudios de Madeira ${ }^{(2)}$ y Cariri (15).

La prueba de correr/caminar de doce minutos, que expresa el desarrollo de la aptitud cardiorrespiratoria, viene a ser uno de los componentes más importantes de la aptitud física orientada a la salud. Los valores del presente estudio revelan un perfil de desempeño con tendencia al aumento de los seis a los diecisiete años de edad en ambos sexos. En los varones, existe una estabilización entre los nueve y los trece años, mientras que en las mujeres esto ocurre entre los once y catorce años de edad. Los resultados son menores a los descritos en los estudios realizados en Madeira (2) y Cariri (15). Las pruebas que valoran la resistencia aeróbica están influenciadas por factores motivacionales y por aspectos relacionados con las dimensiones corporales ${ }^{(1)}$.

Los resultados indican un aumento de los niveles de aptitud física con la edad y verifican la presencia de una amplia variabilidad, las que están en relación con lo descrito en varios estudios longitudinales $(2,12,15,29)$. Resulta importante destacar, a partir de los resultados encontrados en la mayoría de las pruebas, quelas mujeres a partir de los 11-12 años presentan incrementos muy pobres, los cuales estarían reflejando probablemente la interacción de factores biológicos (crecimiento de tejido adiposo, maduración sexual) y culturales como cambios en los intereses sociales, la presión de grupo y falta de motivación para la práctica regular de ejercicio físico ${ }^{(1)}$. Por otro lado, las condiciones diferenciadas del clima, altitud y de infraestructura deportiva que se manifiestan en las tres regiones naturales de donde provienen los datos del estudio podrían explicar la variabilidad interindividual encontrada.

Los valores normativos en términos poblacionales constituyen una herramienta muy importante en el contexto educativo y de salud pública. En el campo educativo debe convertirse en un aliado del profesor y del estudiante para poder identificar con precisión las mejoras en su nivel de aptitud física, de forma que la escuela deberá desempeñar un papel importante en la identificación de escolares en riesgo. En tal sentido, los datos de un niño o adolescente que se localicen por debajo del $\mathrm{P}_{10}$ debería ser tomado como una "alerta" que debe dar lugar a que los encargados de su formación puedan dirigirse a instituciones de salud para que sus profesionales realicen una evaluación con mayor detenimiento y descarten factores que puedan desencadenar enfermedades a futuro.

Es necesario señalar algunos aspectos que limitan el estudio. La primera se refiere a la influencia de la amplia variabilidad del tamaño, composición y forma corporal en el desempeño motor de los niños y adolescentes, que conllevaría a acudir a la Alometría, que desde el punto de vista operacional, pretende corregir el desempeño para diferencias de la morfología externa haciendo uso de modelos lineales o no lineales con efectos aditivos o multiplicativos. La segunda está referida a que la muestra procede de cuatro distritos ubicados en tres zonas geográficamente diferenciadas de la región central del Perú, la cual no permite que sus resultados puedan generalizarse a todo el país; sin embargo, la dimensión de la muestra es elevada y la variabilidad interindividual verificada en cada una de las seis pruebas por sexo e intervalo de edad en cada distrito permite que los valores de referencia propuestos puedan ser utilizados con regularidad. Por último, al ser un estudio transversal, no permite indagar la influencia de otros factores biológicos y socioeconómicos que influirían en el desempeño motor de los niños y adolescentes.

En conclusión, se establecieron las cartas y los valores de referencia específicos por edad y sexo que servirán para la evaluación, interpretación y monitorización de los niveles de aptitud física de niños y adolescentes peruanos. El perfil de las cartas percentílicas presenta un comportamiento similar a otros estudios, y la variabilidad interindividual en ambos sexos en las diversas pruebas motoras es notoria. Se requiere más investigación para establecer puntos de corte validados para todos los componentes de aptitud física asociada a la salud. Este sería un primer esfuerzo que provee valores normativos sobre componentes de aptitud física orientada a la salud en un país que carece de este tipo de información.

\section{AGRADECIMIENTOS}

Los autores agradecen a la Fundación para la Ciencia y la Tecnología de Portugal (SFRH/BD/43305/2008). Nuestro reconocimiento a los profesores y estudiantes de Educación Física de la UNE EGyV que participaron en la recolección de datos. Agradecimiento especial a los niños, adolescentes y sus respectivos profesores que participaron en el Proyecto Crecer con Salud y Esperanza; asimismo, a las autoridades educativas, de salud y políticas de los distritos de Barranco, Junín, La Merced y San Ramón que coadyuvaron para la realización del estudio.

\section{Contribuciones de autoría}

$A B, G B$ y JM participaron en la concepción y el diseño del Proyecto Crecer con Salud y Esperanza. AB realizó el análisis, la interpretación de los datos y la redacción del artículo. JM efectuó la asesoría estadística, la revisión crítica del artículo y la aprobación de la versión final.

\section{Fuentes de financiamiento}

Autofinanciado. 


\section{Conflictos de interés}

Los autores declaran no tener conflictos de interés en la publicación del presente artículo.

\section{REFERENCIAS BIBLIOGRÁFICAS}

1. Malina R, Bouchard C, Bar-Or O. Growth, maturation and physical activity. 4th ed. Champaign: Human Kinetics; 2004.

2. Freitas D, Maia J, Beunen G, Lefevre J, Claessens A, Marques A, et al. Crescimento somático, maturação biológica, aptidão física, actividade física e estatuto socioeconómico de crianças e adolescentes madeirenses. OEstudo de Crescimento da Madeira. Funchal: Universidade da Madeira; 2002.

3. Bouchard C, Shephard RJ. Physical Activity, fitness and health: the model and key concepts. In: Bouchard C, Shephard RJ, Stevens T, editors. Physical activity, fitness, and health: international proceedings and consensus statement. Champaign: Human Kinetics; 1994. p. 77-88.

4. Malina R. Longitudinal perspectives on physical fitness during childhood and youth. In: Classens A, Levefre J, Eynde B (eds). World-wide variation in physical fitness. Leuven: Institute of Physical Education, Catholic University of Leuven; 1993. p. 94-105.

5. Caspersen CJ, Powell KE, Christenson GM. Physical activity, exercise, and physical fitness: definitions and distinctions for health-related research. Public Health Rep. 1985; 100(2):126-31.

6. Ortega FB, Ruiz JR, Castillo MJ, Sjostrom M. Physical fitness in childhood and adolescence: a powerful marker of health. Int J Obes (Lond). 2008; 2(1):1-11.

7. Murphy MH, McNeilly AM, Murtagh EM. Session 1: Public health nutrition: Physical activity prescription for public health. Proc Nutr Soc. 2010;69(1):178-84.

8. Janssen I, LeBlanc AG. Systematic review of the health benefits of physical activity and fitness in school-aged children and youth. Int J Behav Nutr Phys Act. 2010;7:40.

9. Janz KF, Dawson JD, Mahoney LT. Increases in physical fitness during childhood improve cardiovascular health during adolescence: the Muscatine Study. Int J Sports Med. 2002;23 Suppl 1:S15-21.

10. Hasselstrøm H, Hansen SE, Froberg K, Andersen LB. Physical fitness and physical activity during adolescence as predictors of cardiovascular disease risk in young adulthood. Danish youth and sports study. An eight-year follow-up study. Int J Sports Med. 2002; 23 Suppl 1:27-31.

11. American Alliance for Health, Physical Educations, Recreation and Dance (AAHPERD). Health related physical fitness manual. Washington: AAHPERD, 1980.

12. Maia JA, Lopes VP, Bustamante A, Garganta R, Seabra A, Fonseca A, et al. Crescimento e desempenho motor de crianças e jovens Açorianos. Cartas de referência para uso em Educação Física, Desporto, Pediatria e Nutrição. Terceira e Porto: Direcção Regional do Desporto e Faculdade do Desporto; 2007.

13. Pyke JE. The Australian Schools Fitness Test for students aged 7-15: based on the Australian Health and Fitness Survey. Parkside, S. Aust.: Australian Council for Health, Physical Education and Recreation; 1985.

14. Ortega FB, Artero EG, Ruiz JR, España-Romero V, Jiménez-Pavón D, Vicente-Rodríguez G, et al. Physical fitness levels among European adolescents: the HELENA study. Br J Sports Med. 2011;45(1):20-9.

15. Silva S, Beunen G, Maia José. Valores normativos do desempenho motor de crianças e adolescentes: o estudo longitudinal-misto do Cariri. Rev bras educ fís esporte. 2011;25(1):111-25.

16. Alexander P. Aptitud física, características morfológicas, composición corporal. Pruebas estandarizadas en Venezuela, 7.5 a 18.4 años. Caracas: Instituto Nacional de DeportesDepoactión; 1995.

17. Aguilar AC, Pradilla A, Mosquera M, Gracia AB, Ortega JG, Leiva JH, et al. Percentiles de condición física de niños y adolescentes de Santiago de Cali, Colombia. Biomedica. 2011;31(2):242-9.

18. Perú, Ministerio de Educación. Diseño Curricular Nacional de Educación Básica Regular. Lima: MINEDU; 2009.

19. Perú, Ministerio de Educación [Internet]. Lima, Perú; MINEDU; c2009-2010 [citado el 15 de enero del 2012]: Disponible en: http://escale.minedu.gob.pe/magnitudes

20. Prista A, Marques A, Maia J. Relationship between physical activity, socioeconomic status, and physical fitness of 8-15-year-old youth from Mozambique. Am J Hum Biol. 1997;9(4):449-57.

21. Committee of Experts on Sports Research. EUROFIT: Handbook for the EUROFIT tests of physical fitness. 2nd ed. Strasbourg: Committee of Experts on Sports Research; 1993.

22. Welk GJ, Meredith MD. Fitnessgram/activitygram reference guide. Dallas: The Cooper Institute; 2000.

23. Cole TJ, Green PJ. Smoothing reference centile curves: the LMS method and penalized likelihood. Stat Med. 1992;11(10):1305-19.

24. Pan H, Cole TJ. LmsChartMaker Pro version 2.54 , compiled on 21 July 2011. A program for calculating age-related reference centiles using the LMS method. Copyright (C) 2011, Medical Research Council, UK.

25. Pan H, Cole TJ. A comparison of goodness of fi t tests for agerelated reference ranges. Stat Med. 2004;23(11):1749-65.

26. Cole TJ, Green PJ. Smoothing reference centile curves: the LMS method and penalized likelihood. Stat Med. 1992;11(10):1305-19.

27. Royston P, Wright EM. Goodness-of-fit statistics for agespecific reference intervals. Stat Med. 2000;19(21):2943-62.

28. van Buuren S, Fredriks AM. Worm plot: A simple diagnostic device for modeling growth reference curves. Stat Med. 2001;20(8):1259-77.

29. Beunen GP, Simons J. Physical growth, maturation and performance. In: Simons J, Beunen GP, Renson R, Claesens ALM, Vanreusel B, Lefevre JAV, eds. Growth and Fitness of Flemish Girls: The Leuven Growth Study. Champaign, IL: Human Kinetics; 1990. p. 69-118.

30. Sauka M, Priedite IS, Artjuhova L, Larins V, Selga G, Dahlström $\mathrm{O}$, et al. Physical fitness in northern European youth: reference values from the Latvian Physical Health in Youth Study. Scand J Public Health. 2011;39(1):35-43.

Correspondencia: Alcibíades Bustamante Valdivia

Dirección: Rua Plácido Costa 91, 4200.450. Porto, Portugal.

Teléfono: (351) 938405522

Correo electrónico: huanta2609@yahoo.es 


\section{ANEXO 1}

Cuadro 5. Valores de referencia de aptitud física de niños y adolescentes peruanos en edad escolar: Fuerza máxima de extremidad superior, fuerza-resistencia abdominal y fuerza máxima de extremidad inferior.

\begin{tabular}{|c|c|c|c|c|c|c|c|c|c|c|c|c|c|c|}
\hline \multirow{2}{*}{$\begin{array}{l}\text { Edad } \\
\text { (años) }\end{array}$} & \multicolumn{7}{|c|}{ Femenino } & \multicolumn{7}{|c|}{ Masculino } \\
\hline & $P_{3}$ & $\mathbf{P}_{10}$ & $\mathbf{P} 25$ & P50 & P75 & P90 & P97 & $\mathrm{P}_{3}$ & $\mathbf{P}_{10}$ & $\mathbf{P} 25$ & P50 & P75 & P90 & P97 \\
\hline & \multicolumn{14}{|c|}{ Prensión (KgF) } \\
\hline 6 & 4,6 & 5,1 & 5,6 & 6,2 & 6,9 & 7,9 & 9,1 & 4,7 & 5,3 & 6,0 & 6,8 & 7,8 & 8,9 & 10,4 \\
\hline 7 & 5,0 & 5,6 & 6,2 & 7,0 & 8,0 & 9,2 & 10,7 & 5,4 & 6,1 & 6,9 & 8,0 & 9,2 & 10,6 & 12,4 \\
\hline 8 & 5,6 & 6,3 & 7,2 & 8,2 & 9,5 & 11,0 & 12,8 & 6,1 & 7,0 & 8,0 & 9,2 & 10,6 & 12,3 & 14,3 \\
\hline 9 & 6,5 & 7,5 & 8,6 & 9,9 & 11,4 & 13,2 & 15,3 & 7,1 & 8,2 & 9,4 & 10,9 & 12,5 & 14,4 & 16,6 \\
\hline 10 & 7,7 & 8,9 & 10,3 & 11,9 & 13,7 & 15,7 & 18,0 & 8,3 & 9,5 & 10,9 & 12,6 & 14,4 & 16,4 & 18,6 \\
\hline 11 & 9,1 & 10,6 & 12,2 & 14,1 & 16,1 & 18,4 & 20,8 & 9,4 & 10,9 & 12,5 & 14,3 & 16,3 & 18,5 & 20,8 \\
\hline 12 & 10,4 & 12,2 & 14,1 & 16,2 & 18,5 & 20,9 & 23,5 & 10,6 & 12,5 & 14,4 & 16,6 & 18,9 & 21,4 & 24,1 \\
\hline 13 & 11,5 & 13,5 & 15,7 & 18,0 & 20,5 & 23,1 & 25,8 & 12,4 & 14,7 & 17,2 & 19,9 & 22,7 & 25,7 & 28,8 \\
\hline 14 & 12,3 & 14,6 & 16,9 & 19,4 & 22,0 & 24,7 & 27,5 & 15,0 & 17,8 & 20,9 & 24,0 & 27,3 & 30,7 & 34,3 \\
\hline 15 & 13,0 & 15,4 & 17,9 & 20,4 & 23,1 & 25,8 & 28,6 & 18,2 & 21,5 & 25,0 & 28,5 & 32,1 & 35,8 & 39,5 \\
\hline 16 & 13,7 & 16,1 & 18,6 & 21,2 & 23,8 & 26,5 & 29,2 & 21,2 & 24,8 & 28,4 & 32,0 & 35,7 & 39,3 & 42,9 \\
\hline \multirow[t]{2}{*}{17} & 14,3 & 16,7 & 19,2 & 21,8 & 24,4 & 27,0 & 29,6 & 23,7 & 27,3 & 30,9 & 34,4 & 37,9 & 41,4 & 44,8 \\
\hline & \multicolumn{14}{|c|}{ Curl up (rep) } \\
\hline 6 & 5 & 8 & 13 & 19 & 26 & 35 & 46 & 5 & 9 & 14 & 20 & 27 & 36 & 46 \\
\hline 7 & 6 & 10 & 15 & 22 & 31 & 41 & 53 & 6 & 11 & 17 & 24 & 32 & 42 & 53 \\
\hline 8 & 7 & 12 & 18 & 25 & 35 & 46 & 60 & 7 & 13 & 20 & 29 & 38 & 49 & 61 \\
\hline 9 & 8 & 13 & 20 & 28 & 39 & 51 & 65 & 8 & 15 & 24 & 33 & 44 & 55 & 68 \\
\hline 10 & 10 & 15 & 22 & 31 & 42 & 55 & 71 & 9 & 17 & 27 & 38 & 50 & 62 & 75 \\
\hline 11 & 11 & 16 & 24 & 33 & 45 & 59 & 76 & 10 & 20 & 31 & 43 & 55 & 68 & 82 \\
\hline 12 & 12 & 17 & 24 & 34 & 46 & 60 & 78 & 12 & 23 & 35 & 47 & 60 & 73 & 86 \\
\hline 13 & 12 & 18 & 25 & 34 & 45 & 60 & 78 & 14 & 26 & 38 & 50 & 62 & 75 & 88 \\
\hline 14 & 13 & 18 & 25 & 33 & 44 & 58 & 76 & 16 & 28 & 40 & 52 & 64 & 77 & 89 \\
\hline 15 & 15 & 19 & 25 & 33 & 44 & 57 & 75 & 17 & 29 & 41 & 53 & 65 & 77 & 89 \\
\hline 16 & 17 & 21 & 27 & 34 & 44 & 57 & 75 & 18 & 30 & 42 & 54 & 66 & 78 & 90 \\
\hline \multirow[t]{2}{*}{17} & 20 & 24 & 29 & 36 & 45 & 58 & 75 & 18 & 30 & 42 & 54 & 66 & 78 & 90 \\
\hline & \multicolumn{14}{|c|}{ Salto horizontal (cm) } \\
\hline 6 & 50,5 & 60,3 & 70,2 & 80,1 & 90,0 & 100,0 & 110,1 & 60,7 & 71,3 & 81,9 & 92,3 & 102,7 & 113,0 & 123,3 \\
\hline 7 & 58,0 & 68,1 & 78,4 & 88,9 & 99,6 & 110,3 & 121,2 & 67,2 & 78,1 & 89,0 & 99,9 & 110,9 & 121,9 & 133,0 \\
\hline 8 & 66,3 & 76,7 & 87,4 & 98,4 & 109,6 & 121,1 & 132,9 & 74,8 & 85,8 & 97,0 & 108,4 & 120,0 & 131,7 & 143,5 \\
\hline 9 & 74,5 & 84,8 & 95,6 & 106,9 & 118,6 & 130,7 & 143,2 & 81,0 & 92,1 & 103,4 & 115,0 & 126,8 & 138,8 & 151,1 \\
\hline 10 & 81,4 & 91,6 & 102,4 & 113,7 & 125,7 & 138,3 & 151,5 & 86,5 & 97,5 & 108,9 & 120,6 & 132,6 & 145,0 & 157,6 \\
\hline 11 & 86,7 & 96,6 & 107,3 & 118,7 & 130,9 & 144,0 & 157,9 & 91,7 & 102,8 & 114,4 & 126,3 & 138,8 & 151,6 & 164,8 \\
\hline 12 & 89,8 & 99,3 & 109,8 & 121,1 & 133,5 & 146,9 & 161,5 & 95,3 & 106,7 & 118,6 & 130,9 & 143,8 & 157,2 & 171,1 \\
\hline 13 & 91,1 & 100,2 & 110,3 & 121,5 & 133,9 & 147,7 & 163,1 & 99,0 & 110,7 & 123,1 & 136,2 & 149,8 & 164,1 & 179,0 \\
\hline 14 & 91,6 & 100,2 & 109,8 & 120,7 & 133,0 & 147,1 & 163,2 & 104,3 & 116,7 & 129,9 & 144,0 & 158,8 & 174,6 & 191,1 \\
\hline 15 & 92,6 & 100,7 & 109,8 & 120,4 & 132,6 & 146,9 & 163,8 & 111,1 & 124,1 & 138,1 & 153,1 & 169,2 & 186,4 & 204,7 \\
\hline 16 & 94,3 & 101,8 & 110,5 & 120,6 & 132,6 & 146,9 & 164,3 & 115,9 & 129,0 & 143,2 & 158,5 & 175,0 & 192,8 & 211,8 \\
\hline 17 & 95,7 & 102,6 & 110,8 & 120,4 & 131,9 & 145,9 & 163,5 & 118,7 & 131,8 & 146,0 & 161,4 & 178,0 & 196,0 & 215,3 \\
\hline
\end{tabular}

Nota. Artículo disponible en:

Alcibíades Bustamante, Gastón Beunen, José Maia. VALORACIÓN DE LA APTITUD FíSICA EN NIÑOS Y ADOLESCENTES:

CONSTRUCCIÓN DE CARTAS PERCENTÍLICAS PARA LA REGIÓN CENTRAL DEL PERÚ. Rev Peru Med Exp Salud Publica. 2012; 29(2):188-97. 
Cuadro 6. Valores de referencia de aptitud física de niños y adolescentes peruanos en edad escolar: Flexibilidad, resistencia cardiorespiratoria y agilidad.

\begin{tabular}{|c|c|c|c|c|c|c|c|c|c|c|c|c|c|c|}
\hline \multirow{2}{*}{$\begin{array}{l}\text { Edad } \\
\text { (años) }\end{array}$} & \multicolumn{7}{|c|}{ Femenino } & \multicolumn{7}{|c|}{ Masculino } \\
\hline & $P_{3}$ & $P_{10}$ & $\mathbf{P} 25$ & $P_{50}$ & P75 & P90 & P97 & $\mathbf{P}_{3}$ & $\mathbf{P}_{10}$ & $\mathbf{P} 25$ & P50 & P75 & P90 & P97 \\
\hline & \multicolumn{14}{|c|}{ Sentar y alcanzar $(\mathrm{cm})$} \\
\hline 6 & 16,3 & 19,2 & 21,7 & 24,0 & 26,0 & 27,9 & 29,6 & 14,7 & 17,5 & 20,2 & 22,9 & 25,5 & 28,1 & 30,6 \\
\hline 7 & 15,3 & 18,5 & 21,2 & 23,6 & 25,7 & 27,7 & 29,6 & 14,4 & 17,1 & 19,8 & 22,4 & 25,0 & 27,5 & 30,0 \\
\hline 8 & 14,4 & 17,8 & 20,6 & 23,2 & 25,5 & 27,6 & 29,6 & 14,0 & 16,8 & 19,4 & 21,9 & 24,4 & 26,9 & 29,3 \\
\hline 9 & 13,6 & 17,1 & 20,2 & 22,8 & 25,3 & 27,5 & 29,6 & 13,7 & 16,4 & 18,9 & 21,4 & 23,8 & 26,2 & 28,5 \\
\hline 10 & 13,1 & 16,7 & 19,8 & 22,6 & 25,1 & 27,5 & 29,7 & 13,3 & 16,0 & 18,5 & 20,9 & 23,2 & 25,5 & 27,8 \\
\hline 11 & 12,8 & 16,4 & 19,6 & 22,4 & 25,0 & 27,5 & 29,8 & 13,1 & 15,6 & 18,1 & 20,5 & 22,8 & 25,0 & 27,2 \\
\hline 12 & 12,8 & 16,4 & 19,5 & 22,4 & 25,1 & 27,6 & 30,0 & 13,0 & 15,5 & 18,0 & 20,4 & 22,6 & 24,8 & 27,0 \\
\hline 13 & 13,0 & 16,4 & 19,5 & 22,4 & 25,1 & 27,7 & 30,2 & 13,1 & 15,7 & 18,2 & 20,6 & 22,8 & 25,0 & 27,2 \\
\hline 14 & 13,2 & 16,6 & 19,7 & 22,6 & 25,3 & 27,9 & 30,5 & 13,6 & 16,2 & 18,8 & 21,2 & 23,5 & 25,7 & 27,9 \\
\hline 15 & 13,6 & 16,9 & 19,9 & 22,9 & 25,7 & 28,4 & 31,0 & 14,3 & 17,0 & 19,6 & 22,0 & 24,4 & 26,7 & 28,8 \\
\hline 16 & 14,1 & 17,4 & 20,5 & 23,4 & 26,3 & 29,1 & 31,9 & 15,1 & 17,9 & 20,5 & 23,0 & 25,4 & 27,7 & 29,9 \\
\hline 17 & 14,7 & 18,0 & 21,1 & 24,2 & 27,2 & 30,1 & 33,0 & 16,0 & 18,8 & 21,5 & 24,0 & 26,3 & 28,6 & 30,9 \\
\hline \multicolumn{15}{|c|}{ Correr/caminar de 12 minutos (mts) } \\
\hline 6 & 722 & 808 & 910 & 1030 & 1173 & 1345 & 1553 & 1170 & 1308 & 1428 & 1535 & 1632 & 1744 & 1844 \\
\hline 7 & 793 & 888 & 999 & 1131 & 1290 & 1482 & 1716 & 1190 & 1338 & 1471 & 1592 & 1704 & 1819 & 1928 \\
\hline 8 & 857 & 958 & 1078 & 1221 & 1394 & 1604 & 1863 & 1208 & 1359 & 1500 & 1630 & 1755 & 1872 & 1994 \\
\hline 9 & 904 & 1010 & 1136 & 1286 & 1469 & 1693 & 1972 & 1216 & 1363 & 1507 & 1644 & 1777 & 1911 & 2042 \\
\hline 10 & 939 & 1048 & 1178 & 1334 & 1524 & 1759 & 2055 & 1227 & 1369 & 1510 & 1650 & 1791 & 1934 & 2082 \\
\hline 11 & 959 & 1069 & 1200 & 1359 & 1553 & 1795 & 2101 & 1248 & 1380 & 1517 & 1660 & 1806 & 1957 & 2112 \\
\hline 12 & 962 & 1071 & 1202 & 1360 & 1554 & 1797 & 2107 & 1272 & 1402 & 1538 & 1682 & 1832 & 1989 & 2142 \\
\hline 13 & 966 & 1074 & 1203 & 1360 & 1554 & 1797 & 2109 & 1311 & 1440 & 1575 & 1716 & 1864 & 2019 & 2171 \\
\hline 14 & 985 & 1093 & 1222 & 1379 & 1573 & 1818 & 2135 & 1351 & 1481 & 1615 & 1755 & 1900 & 2049 & 2201 \\
\hline 15 & 1017 & 1125 & 1256 & 1414 & 1611 & 1859 & 2181 & 1390 & 1524 & 1660 & 1799 & 1940 & 2084 & 2231 \\
\hline 16 & 1047 & 1156 & 1287 & 1446 & 1643 & 1892 & 2217 & 1449 & 1591 & 1733 & 1874 & 2015 & 2156 & 2297 \\
\hline \multirow[t]{3}{*}{17} & 1073 & 1181 & 1311 & 1469 & 1665 & 1913 & 2237 & 1525 & 1680 & 1831 & 1977 & 2120 & 2260 & 2398 \\
\hline & \multicolumn{14}{|c|}{ Va y viene (seg) } \\
\hline & P97 & P90 & P75 & P50 & $\mathbf{P 2 5}$ & $P_{10}$ & $\mathbf{P}_{3}$ & P97 & P90 & P75 & P50 & $\mathrm{P} 25$ & $\mathbf{P}_{10}$ & $\mathbf{P}_{3}$ \\
\hline 6 & 23,2 & 24,5 & 25,9 & 27,5 & 29,2 & 30,9 & 32,9 & 22,3 & 23,6 & 24,9 & 26,3 & 27,7 & 29,3 & 31,0 \\
\hline 7 & 22,2 & 23,5 & 25,0 & 26,5 & 28,2 & 30,0 & 32,0 & 21,2 & 22,6 & 24,0 & 25,6 & 27,2 & 28,9 & 30,8 \\
\hline 8 & 21,2 & 22,6 & 24,1 & 25,6 & 27,3 & 29,1 & 31,1 & 20,0 & 21,4 & 22,9 & 24,6 & 26,3 & 28,2 & 30,2 \\
\hline 9 & 20,4 & 21,8 & 23,3 & 24,9 & 26,6 & 28,4 & 30,3 & 19,1 & 20,6 & 22,1 & 23,7 & 25,4 & 27,2 & 29,2 \\
\hline 10 & 19,9 & 21,3 & 22,8 & 24,4 & 26,0 & 27,8 & 29,6 & 18,6 & 20,0 & 21,4 & 23,0 & 24,6 & 26,3 & 28,2 \\
\hline 11 & 19,7 & 21,1 & 22,5 & 24,1 & 25,7 & 27,4 & 29,2 & 18,2 & 19,6 & 21,0 & 22,5 & 24,0 & 25,6 & 27,3 \\
\hline 12 & 19,7 & 21,0 & 22,5 & 24,0 & 25,5 & 27,2 & 28,9 & 17,9 & 19,3 & 20,7 & 22,1 & 23,5 & 25,0 & 26,5 \\
\hline 13 & 19,7 & 21,1 & 22,5 & 23,9 & 25,5 & 27,0 & 28,6 & 17,8 & 19,1 & 20,4 & 21,8 & 23,1 & 24,5 & 25,8 \\
\hline 14 & 19,8 & 21,2 & 22,5 & 23,9 & 25,4 & 26,9 & 28,4 & 17,5 & 18,8 & 20,1 & 21,4 & 22,6 & 23,8 & 25,0 \\
\hline 15 & 19,9 & 21,2 & 22,5 & 23,8 & 25,2 & 26,6 & 28,0 & 17,2 & 18,5 & 19,7 & 20,8 & 21,9 & 23,0 & 24,0 \\
\hline 16 & 19,8 & 21,1 & 22,3 & 23,6 & 24,8 & 26,1 & 27,4 & 17,0 & 18,2 & 19,3 & 20,4 & 21,4 & 22,3 & 23,2 \\
\hline 17 & 19,9 & 21,0 & 22,1 & 23,2 & 24,4 & 25,5 & 26,7 & 16,8 & 18,0 & 19,1 & 20,1 & 21,1 & 22,0 & 22,8 \\
\hline
\end{tabular}

Nota. Artículo disponible en:

Alcibíades Bustamante, Gastón Beunen, José Maia. VALORACIÓN DE LA APTITUD FíSICA EN NIÑOS Y ADOLESCENTES:

CONSTRUCCIÓN DE CARTAS PERCENTÍLICAS PARA LA REGIÓN CENTRAL DEL PERÚ. Rev Peru Med Exp Salud Publica. 2012; 29(2):188-97. 